\title{
Design, optimization, and testing of a high-speed centrifugal pump for motorsport application
}

\author{
Luigi Mariani $^{1 *}$, Giammarco Di Giovine ${ }^{1}$, Davide Di Battista ${ }^{1}$, and Roberto Cipollone ${ }^{1}$ \\ ${ }^{1}$ Dipartimento di Ingegneria Industriale e dell'Informazione e di Economia, Università degli Studi \\ dell'Aquila, P. le Pontieri, 1 - Monteluco di Roio, 67100, L'Aquila, Italy
}

\begin{abstract}
Centrifugal pumps are designed to have their BEP (Best Efficiency Point) for a given flow rate, hydraulic head, and speed. In the design phase, those parameters are combined into a dimensionless specific speed used to define geometry of the pump.

In this paper, a downsized centrifugal pump has been designed to have high efficiency at very high speeds (10000 - 15000 RPM), as requested by the cooling circuit of an engine for motorsport and racing applications. The pump design point was $13 \mathrm{~L} / \mathrm{min}$ and 3.0 bar at $12000 \mathrm{RPM}$, while the impeller external diameter is $34 \mathrm{~mm}$.

A mathematical model has been realized to optimize the pump in the early design phase through an iterative process, based on a 0D procedure which generates the optimal geometry of both impeller and volute. Hence, the model estimates main losses and, thus, hydraulic, volumetric, and organic efficiency. Once the geometry is generated, the performance of the pump has been verified on the design working point through a detailed CFD analysis. Physical phenomena that occur when the pump is running have been simulated, to represent as closely as possible vein fluid detachments, cavitation, and backflow at clearances between impeller and pump casing. At last, a prototype of the pump has been built and experimentally characterized in a dynamic test bench able to reproduce the characteristic curves (hydraulic head and efficiency) at very high revolution speeds as well as the performances in real time-varying operational conditions.
\end{abstract}

\section{Introduction}

Centrifugal pumps are one of the most important equipment in all sectors. They are widely used in residential applications, for thermal and cooling plants and household electric devices, or in ORC based micro-cogeneration systems [1], in industrial sectors for applications which involve many different needs and the compliance with several fluids and specifics [2]: in few words, pumps are used to move fluids for a number of services.

An important sector of application is the cooling sector of internal combustion engines for on-the-road transportation, where through a suitable design they are participating to the transition toward more sustainable mobility [3]. In fact, they are usually present in conventional vehicle for engine cooling and lubrication needs, but significant focusing is

\footnotetext{
${ }^{*}$ Corresponding author: luigi.mariani@graduate.univaq.it
} 
deserving for hybrid, electric and hydrogen vehicles, where the need to control the operating temperature of batteries, electric motor/generator, electronic devices and fuel cells is pushing the research towards unexplored devices, like for instance volumetric pumps, with novel driving and control strategies $[3,4]$. For instance, the need to fulfill contemporaneously different thermal needs led to the development of hydraulic circuits operating in parallel within the same engine bay, operating at different temperature levels $[5,6]$. In this way, engine head and block can have a separated cooling circuit, in order to increase engine efficiency [7], charge air can be water cooled, enhancing engine cylinder filling [8] and leading to highly downsized engine [9]. Also, the cabin air conditioning can benefit of a liquid cooled condenser [10] placed on the low temperature cooling circuit. Furthermore, battery cooling and heating are essential for electric cars since it highly affects the charging and discharging processes; in fact, the amount of heat generation due to battery internal chemical reactions and ohmic internal resistance causes a battery temperature to rise [11]; battery heating is important as well during cold conditions in order to save efficiency and life. In fuel cell-based vehicle, the conventional PEMFC needs to operate strictly at lowtemperature with humidification of the membrane and secondary water circuits are mandatory [12]. Temperature of the on-board electronics must be strictly under control, and this requires an auxiliary cooling circuit. Moreover, the optimization of cooling water system is important to improve the energy efficiency and economic performance of industrial systems; thus, a geometrical optimization of pump can save energy cost and simplify the network structure [13].

Most part of these applications require low and very low flow rates and limited pressure head: this happens for electric and hybrid cars as well as for conventionally fueled vehicles in which cooling water circulation is reduced and additional thermal needs are fulfilled with specific and separate circuits (cabin heating/cooling, charge air \& oil cooling, etc.). Moreover, the most used drive for these auxiliary and ancillary pumps is of electrical type, for increased flexibility and operability management. This is truer for hybrid and electric vehicles, where the higher electrification grade facilitates the introduction of electric subsystems. In any case, they should have a very low impact in terms of weight, space requirements and overall power absorbed.

Several of these requirements and specifications are more important for racing and motorsport applications, where every little improvement is desirable; concerning the pumps, a specific research is oriented toward very high operating speed leading to high powerdensity pumps, including their driving device. In this way, size and weight of the pump can be reduced. Moreover, being operating rotational speeds quite high, the electric motor which drives the pump can be downsized.

The traditional procedure used to design a centrifugal pump starts with the definition of its specific speed, which accounts for desired pressure head, required flow rate and revolution speed (design point at Best Efficiency Point, BEP). Then, inlet and outlet speed triangles are calculated to draw the complete blade profile and to evaluate the work exchanged between fluid and machine, following the simplified Euler's equation. At last, volute profile and transversal sections are calculated through the conservation of angular momentum or assuming a decrease of it following the flow due to losses.

High specific speed pumps have been studied in the literature for their intrinsic difficulties to be designed, optimizing their performance in off-design conditions with a high geometrical ratio between constructive gap and blade height [14]. Semi-open and open impeller have been proposed, to reduce the axial forces on the pump rotor $[14,15]$. Moreover, one of the most important issue associated with high-speed pumps is the cavitation phenomena [16] due to the low-pressure level the pump can suffer at impeller inlet; static components at the pump inlet, like pre-swirl or inducers are used in order to reduce the fluid vaporization. Also, the blade number has been deserving attention to optimize pump performance; it could be 
concluded that the performance of centrifugal pump tends to be more sensitive to the change in the revolution speed rather than the change in the number of blades [17]. It is known that the shape of the volute in centrifugal pumps is fundamental to recover pressure levels from kinetic energy: this is challenging in high-speed pumps where the very high fluid speed leaving the impeller induces fluid detachment and, thus, hydrodynamic and unpredictable losses. The influence of the volute geometry plays another key-role both for noise and vibrations [18].

Recently, 3D numerical analysis is widely used in the study of centrifugal pumps, in order to clearly represent physical phenomena and, thus, to predict pump performance. In the most common approach, the fluid domain is split into a static and a rotating part, with bounded matching fluid-fluid interfaces, in a steady state analysis (frozen rotor approach). The standard k- $\varepsilon$ method is usually chosen to calculate the turbulent flow field into the machine, and to simulate properly the effect of roughness near static and moving walls [19].

Moreover, the grid specifications deserve particular care, because the number of elements is proportional to time consumption and computational cost. The tetrahedral unstructured mesh is commonly used where the geometry of the fluid domain has a complex shape; instead, the sweep structured mesh with inflation is strictly recommended when the domain is regular, for instance on cylindrical connecting pipes [20].

In this paper, a design and optimization procedure of a high-speed low-flow centrifugal pump is presented, in order to achieve high efficiency and reduce its impact in terms of power absorbed, weight and encumbrances for an automotive application, particularly desired in the racing environment. Main constraint was referred to the dimension of the pump related to the space available on board. So, the radial dimension of the pump (impeller and expected volute) was almost fixed resulting preliminary defined the range of the revolution speed at the design point. The resulting value was not constrained by a mechanical linkage with the crankshaft being the actuation of the pump done by a properly designed electric motor. Inside these constraints, the more suitable design way was to make an initial reference to a $0 \mathrm{D}$ model developed by the Authors, subsequently refined by a 3D approach. This step allowed to evaluate the volumetric efficiency of the pump (which was expected lower than conventional designs due to the reduced dimension of the pump) and the occurrence of cavitation expected from the high revolutions speed, in the range of $10000-15000$ RPM. So, a mathematical procedure is developed through an iterative process: it is based on a $0 \mathrm{D}$ model, which considers the geometrical features related to the velocity of the fluid in the different main sections (impeller inlet, impeller outlet and volute), assessing the velocity streamline and hydraulic efficiency, without the need of high computational resources related to CFD analyses. Hence, the model calculates the optimal impeller angles to establish the correct value of outer speed, and generates the geometry of the volute, in terms of spiral radiuses and flow area evolution, by applying the free vortex law. Once the geometry is generated, the performances of the pump have been verified on the design working point through a dedicated CFD analysis.

A pump prototype has been built and characterized in a test bench [21] able to reproduce the operating very high revolution speeds (12000 RPM).

\section{Pump design}

In this study, design specifications imposed by the circuit are $13 \mathrm{~L} / \mathrm{min}$ and $3.0 \mathrm{bar}$. To reduce the outer impeller diameter $\left(\mathrm{D}_{\mathrm{EXT}}\right)$, a complete study on the revolution speed of the pump has been carried out (Fig. 1). In the x-axis of the plot, rotational speed has been reported in the most common unit of measure in this kind of application (RPM), while $n_{s}$ has been calculated considering the following unit of measures: $\mathrm{rad} / \mathrm{s}$ for $(\omega), \mathrm{m}^{3} / \mathrm{s}$ for $(\mathrm{Q})$, and $\mathrm{J} / \mathrm{kg}$ for $(\mathrm{gH})$. The impeller external diameter has been calculated based on the affinity law (Eq. (1)), which 
establishes the dependence between flow rate, diameter, and rotational speed. By fixing flow rate, it shows how diameter is influenced by modifying pump rotational speed. When the pump design speed increases, specific rotational speed $\left(\mathrm{n}_{\mathrm{S}}\right)$ increase too, while the diameter reduces with decreasing slope. This invites designers to set up a revolution speed which is a trade-off between decreasing diameter and revolution speed increase. Thus, 12000 RPM has been chosen as the design rotational speed, in order to reach a sensible reduction of the dimensions of the pump which is requested by the specific application.

$$
\frac{\mathrm{Q}_{2}}{\mathrm{Q}_{1}}=\frac{\mathrm{D}_{2}}{\mathrm{D}_{1}} \frac{\mathrm{N}_{2}}{\mathrm{~N}_{1}}
$$

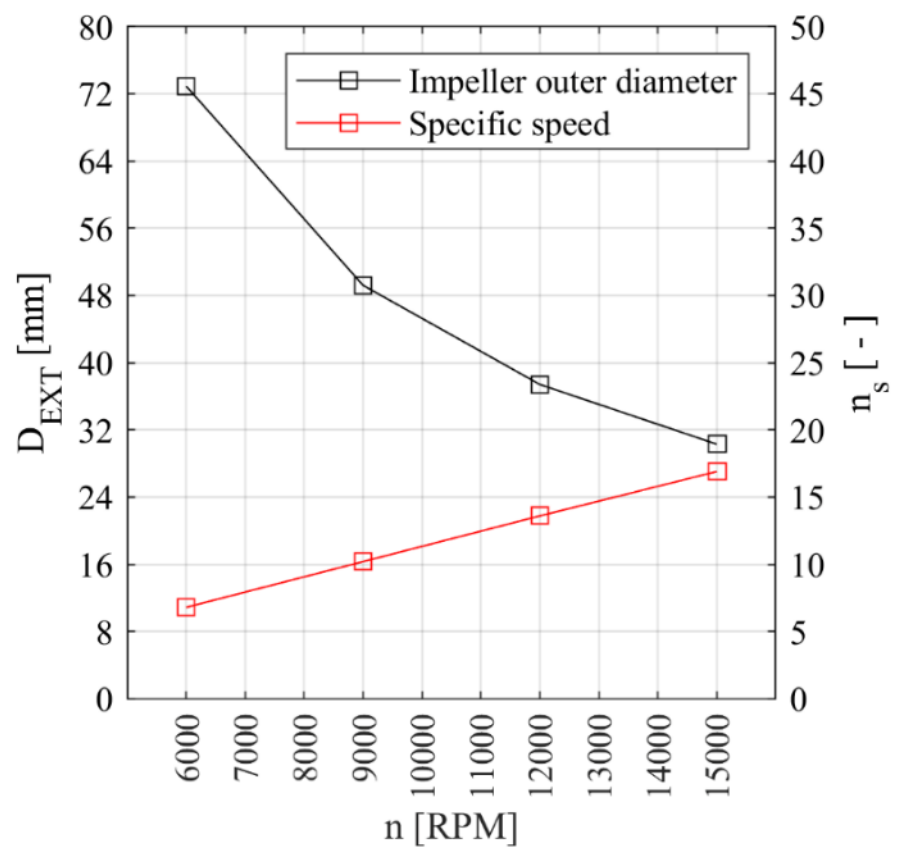

Fig. 1. Impeller outer diameter and specific speed dependence on design rotational speed.

After having fixed the design revolution speed, being known flow rate and pressure delivered, the complete geometry of the pump has been generated through a simple 0D design procedure, taken from [25] and implemented with a proprietary script in Matlab ${ }^{\circledR}$. The proposed algorithm generates the impeller hub and shroud profiles, using a polynomial second order Bezier curve, with three control points [22, 23]. In Fig. 2 the implemented meridian section of the pump has been reported as well as main variables calculated by the procedure. 


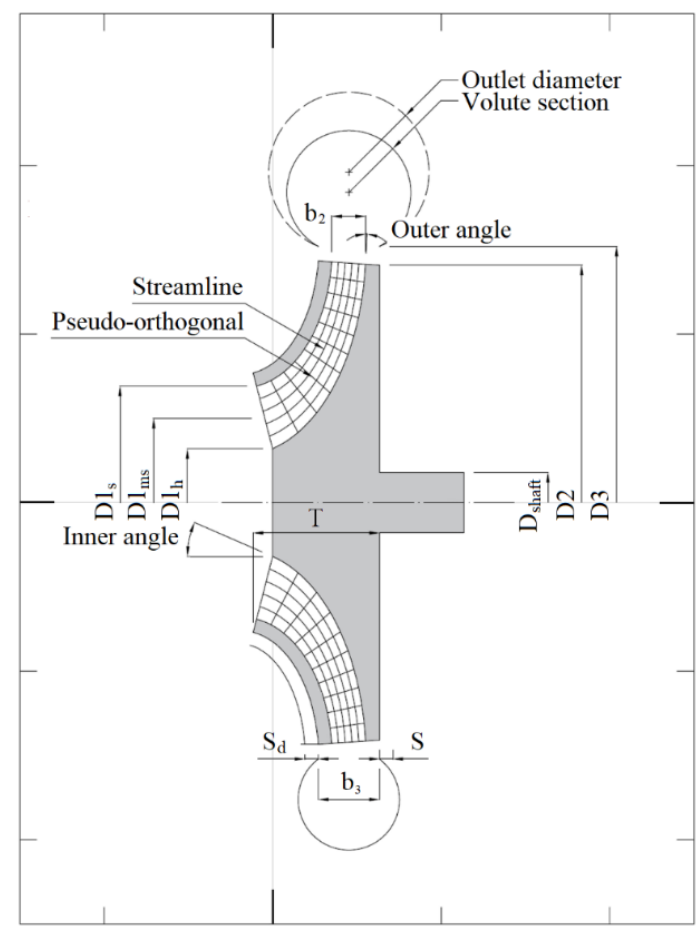

Fig. 2. Impeller meridian section as represented in the mathematical model.

The main dimensions calculated are the impeller inner, mean, and outer diameters, the volute sections, the hub diameter, and the impeller inlet and outlet angles. Assuming a linear variation between blade inlet and outlet angles, the full geometry of the blade can be calculated by geometrical considerations; the cross sections of the impeller passages have been fully determined. Subsequently, the mathematical model estimates pump performances. The impeller duct is divided into a regular grid, composed by streamlines and pseudoorthogonal lines. On the resulting nodes, the mathematical model calculates velocity and pressure applying continuity and angular momentum conservation equations under the hypotheses of ideal flow. On this initial base, hydraulic, volumetric, and viscous losses are calculated. An iterative process is needed in order to converge toward a stabilized hydraulic efficiency [24].

In Fig. 3 the structure of the mathematical model is posted. The code maximizes the volumetric efficiency, varying iteratively the dimensions of impeller hub and pump casing, and runs a complete cycle initialized by a first attempt value imposed as an input displacement. A minimum volumetric efficiency is set in input and, thus, a first geometry of the pump is generated. The sub-cycle that calculates the volumetric efficiency is inserted into the main cycle, giving the hydraulic efficiency. 


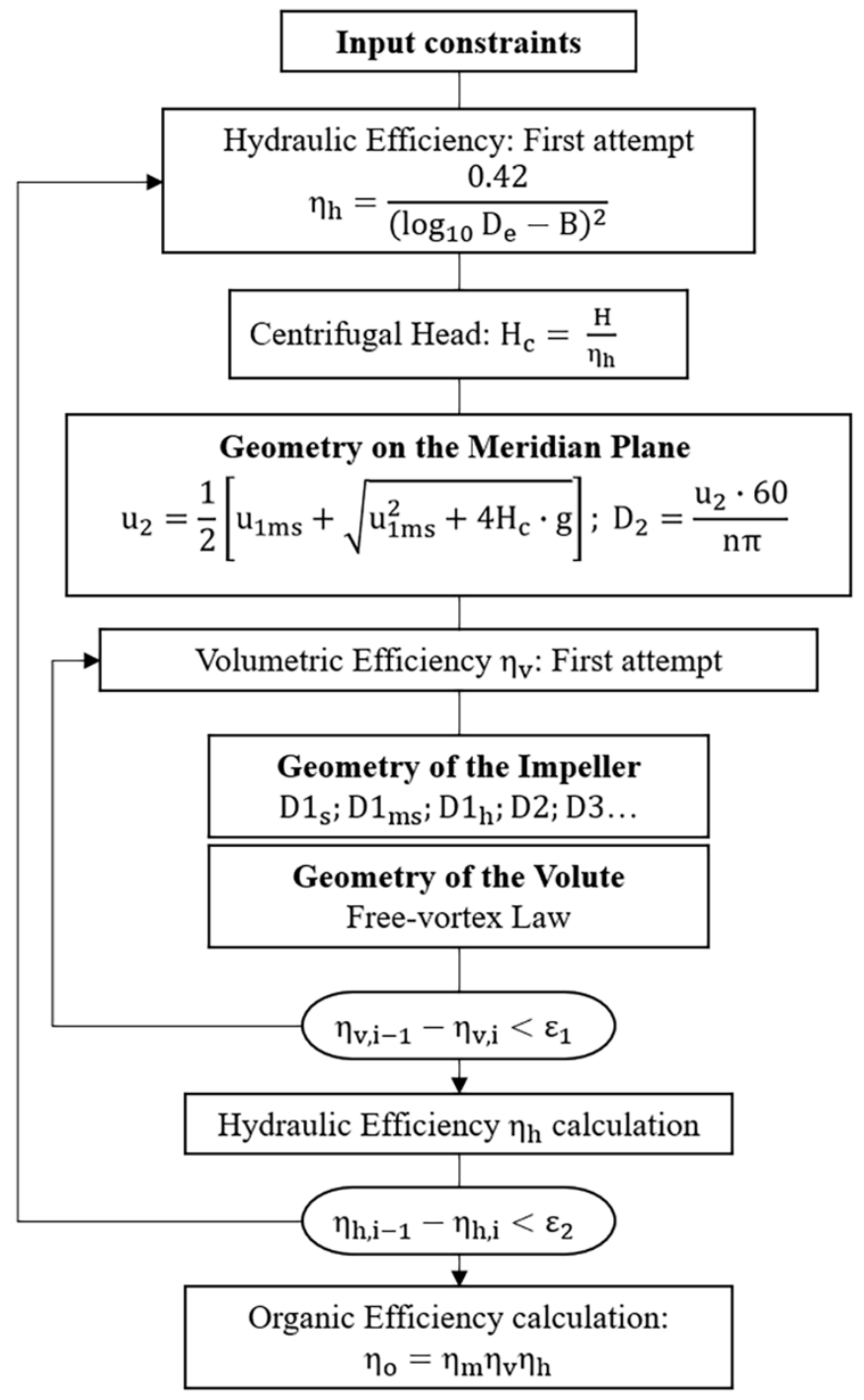

Fig. 3. Structure of the mathematical model implemented in Matlab®.

The geometry of the impeller is generated on the meridian plane, by calculating the outer blade velocity $\mathrm{u}_{2}$ and the outer diameter $\mathrm{D}_{2}$ (Fig. 4). The outer blade angle is defined in order to minimize fluid dynamic losses, and to avoid vein fluid detachments. Once the absolute velocity at the impeller outer section is known, the code generates the complete geometry of the volute. The angular momentum of the fluid increases following the spiral axis of the volute because the distance from the center of rotation increases too. Thus, at every step angle, the area of passage increases, in accordance with the free-vortex law. The angle step adopted for the calculation is 10 degrees.

All input parameters used for the calculation are reported in Table 1. They act as constraints for the mathematical model, in terms of final dimensions and performances of the pump. 
Table 1. Input parameters of the script implemented in Matlab®.

\begin{tabular}{llr}
\hline \multicolumn{3}{c}{ Input Parameters } \\
\hline Inlet diameter [m] & $\mathrm{D}_{1 \mathrm{~s}}$ & 0.012 \\
Rotational speed [RPM] & $\mathrm{n}$ & 12000 \\
Flow rate [L/min] & $\mathrm{Q}$ & 13 \\
Hydraulic head [m] & $\mathrm{H}$ & 30.7 \\
Number of blades & $\mathrm{z}$ & 7 \\
Shaft diameter [m] & $\mathrm{D}_{\mathrm{s}}$ & 0.003 \\
Outlet diameter [m] & $\mathrm{D}_{\mathrm{m}}$ & 0.01 \\
Outer blade height [m] & $\mathrm{b}_{2}$ & 0.0018 \\
Centrifugal vs. real head & $\mathrm{H}_{\mathrm{c}}$ & 1.1 \\
\cline { 2 - 3 } Volute areas & $\mathrm{H}$ & \\
Number of streamlines & $\mathrm{Circular}$ & \\
Number of pseudo-orthogonal lines & - & 12 \\
Impeller/stator gap [m] & - & 0.001 \\
Kinematic viscosity [m $\left.{ }^{2} / \mathrm{s}\right]$ & $\mathrm{S}_{\mathrm{d}}$ & $1.2 \mathrm{e}-06$ \\
Roughness [m] & $\mathrm{N}$ & $5.0 \mathrm{e}-05$ \\
Blade inlet thickness [m] & $\Delta$ & 0.0005 \\
Blade outlet thickness [m] & $\mathrm{t}_{1}$ & 0.0005 \\
Minimum volumetric efficiency & $\mathrm{t}_{2}$ & 0.6 \\
Estimated mechanical efficiency & $\eta_{\mathrm{v}}$ & 0.87 \\
\hline
\end{tabular}

Once the design iterative procedure is over, the model gives the list of the principal dimensions of the designed pump. It sums up fixed and calculated values, and contains hub and shroud angles, blade heights, volute diameters and spiral radiuses. The efficiency of the pump has been calculated considering the empirical relationships reported in [25], and taking into account mechanical, disk friction, volumetric and hydraulic losses.

Implementing the procedure, the full geometry of the pump has been obtained. It is posted in Fig. 4, with two part-bodies, pump casing, and impeller.
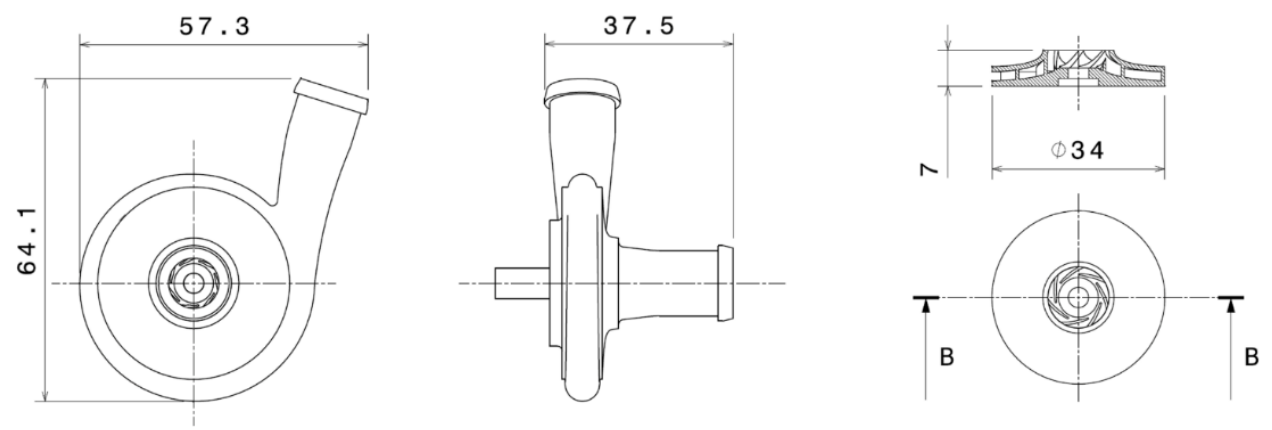

Fig. 4. Impeller and volute geometry (values are in $\mathrm{mm}$ ).

Although mechanical, volumetric, and hydraulic losses are estimated, the design procedure calculates theoretical values, and the $0 \mathrm{D}$ flow does not offer a real representation 
of physical phenomena that take place inside the pump. Hence, the performances of the pump at the design working point have been evaluated through a CFD analysis.

\section{CFD model}

CFD approach has been carried out to analyze the flow field behavior both in the impeller and in the volute using the Ansys ${ }^{\circledR}$ Fluent software. Reynolds-averaged Navier-Stokes's 3D equations have been solved, with the Finite Volume Method (FVM), with a frozen rotor simulation. A k- $\omega$ SST has been used as turbulence model. It combines the original Wilcox model [25] for a near-wall use and standard k- $\varepsilon$ model away from walls with a blending function.

Boundary conditions are total pressure at pump inlet and mass flow rate at pump outlet. The other surfaces have been considered as wall boundary conditions, with static wall for pump casing surfaces and rotating wall at 12000 RPM for impeller blades, hub, and shroud. No slip wall condition has been applied.

Fig. 5 shows the results of the simulation in terms of pressure contour in the frontal section. It is evident how the high revolution speed causes regions of at the impeller inlet characterized by pressure values below the atmospheric datum, till to $0.5-0.55$ bar. Cavitation will occur certainly without a suitable pressurization at inlet insured by a pressurization tank. It is evident also a slight pressurization inside the impeller passages and, finally, a sensible pressure increases when the fluid runs inside the volute, reaching a value of about 3.6 bar at pump outlet.
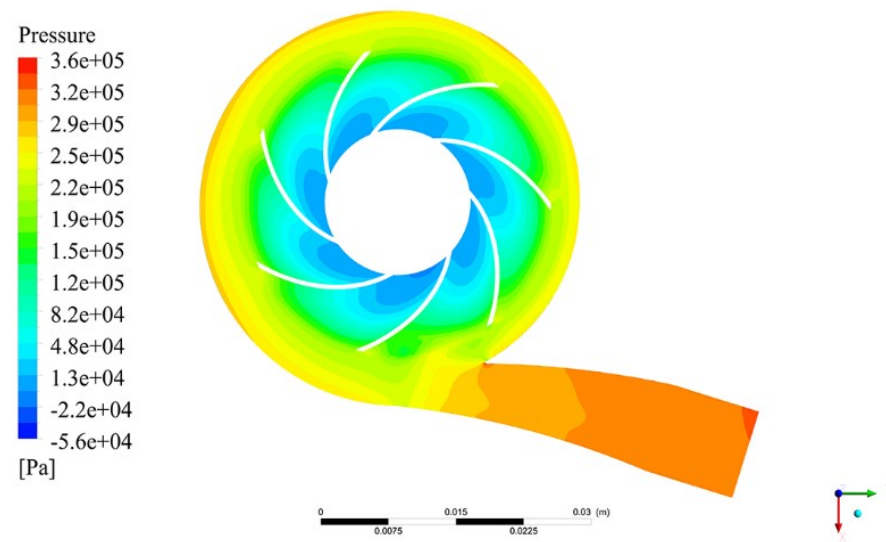

Fig. 5. Pressure contours at blade-to-blade plane.

In Fig. 6 a clearer situation at impeller inlet is shown concerning the occurrence of cavitation. Through the CFD analysis, pressure levels at impeller blade have been evaluated, to consider the possibility that local cavitation phenomena can occur. In fact, if the pressure of the fluid at the impeller inlet decreases under the saturation pressure at that temperature, fluid evaporates, and cavitation takes place. When the fluid enters the impeller vanes, it has a heavy acceleration and pressure quickly decreases. Pressure decreases as well due to the losses occurring when the fluid approaches and enters the high-speed rotating ducts. These fluid dynamic conditions become critical if the fluid reaches the saturation pressure at the inlet fluid temperature and cavitation takes place. It is important to remark that this cavitation occurrence was expected due to the very high revolution speed, widely outside of the conventional pump operating ranges. Fig. 6 refers on the quantitative aspect (and geometrical extension) of cavitation, inviting to a specific pressurization of the hydraulic circuit: the 3D calculation puts in evidence a minimum pressure value about $56 \mathrm{kPa}$ below the atmospheric 
datum which was fixed at pump inlet. This vacuum degree can be easily recovered pressurizing the circuit at pump inlet, also gaining a greater margin in the case of higher revolution speeds.

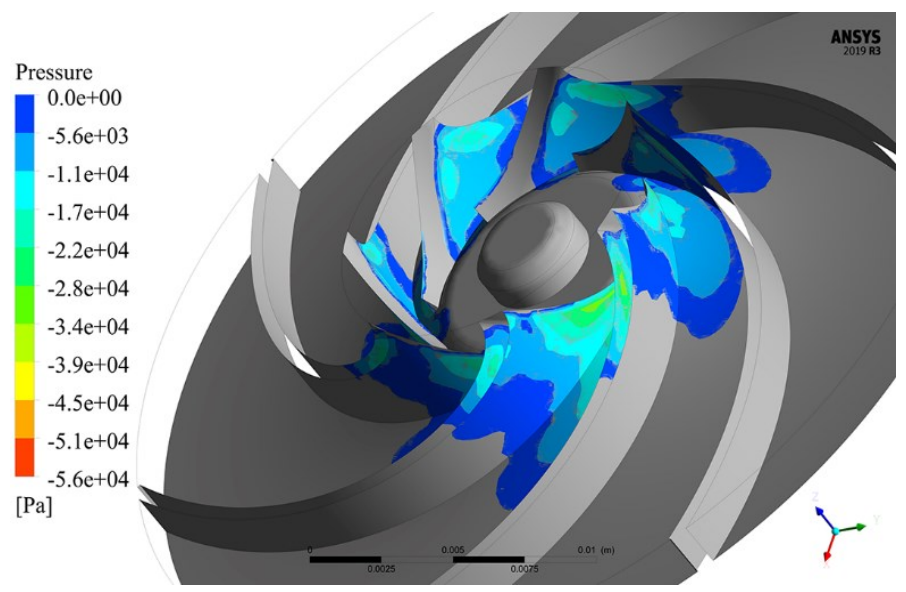

Fig. 6. Pressure zones at impeller domain.

In Fig. 7 a complete representation of the leakage flows at the clearances between impeller and pump casing is shown on the meridian plane. This physical phenomenon is due to the difference of the pressure level between the impeller inlet and outlet; thus, a backflow occurs.

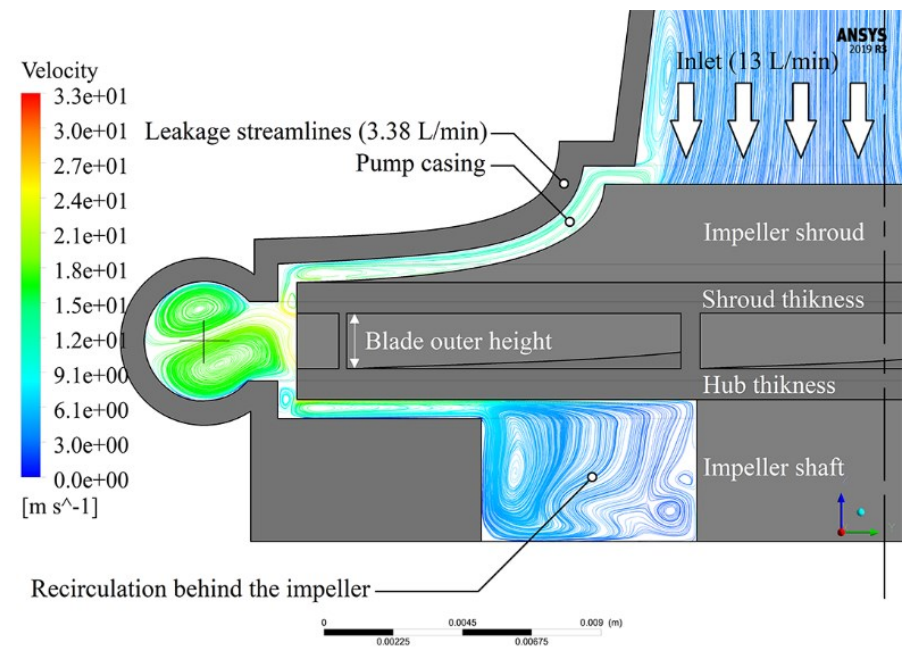

Fig. 7. Focus on the meridian plane of the pump. A backflow of $3.38 \mathrm{~L} / \mathrm{min}$ occurs at clearance between impeller and pump casing, while an overall flow rate of $13 \mathrm{~L} / \mathrm{min}$ is delivered by the pump. A recirculation takes place behind the impeller, where the seal is installed.

The complete output of CFD simulation considered for the evaluation of the performances of the pump have been reported in Table 2. The calculated pressure rise is higher to the design one, and the relative error is about 7.7\%; this gap can be associated to the surface roughness imposed at CFD pre-processing phase, that is $50 \mu \mathrm{m}$. By increasing the value of roughness, fluid dynamic losses increase and, thus, overall pressure rise generated by the 
pump decreases. So, the relative error of $7.7 \%$ decreases and the results becomes more precise. Volumetric efficiency is lower than common values associated with centrifugal pumps, because of the small size of the pump; in fact, technological issues force to guarantee a minimum gap between pump casing and impeller, that becomes relevant in percentage to the dimensions of the pump, in particular to the blade outer height.

Table 2. CFD calculation results.

\begin{tabular}{|c|c|c|}
\hline \multicolumn{3}{|l|}{ Direct results } \\
\hline Inlet mean pressure $[\mathrm{Pa}]$ & $\mathrm{p}_{1}$ & $-3.05 \mathrm{e}+01$ \\
\hline Outlet mean pressure $[\mathrm{Pa}]$ & $\mathrm{p}_{2}$ & $3.23 \mathrm{e}+05$ \\
\hline Inlet mean absolute velocity $[\mathrm{m} / \mathrm{s}]$ & $c_{1}$ & 2.77 \\
\hline Outlet mean absolute velocity $[\mathrm{m} / \mathrm{s}]$ & $\mathrm{c}_{2}$ & 3.51 \\
\hline Fluid dynamic torque $[\mathrm{Nm}]$ & $\mathrm{C}$ & 0.14 \\
\hline \multicolumn{3}{|c|}{ Indirect results } \\
\hline Pressure raise [bar] & $\Delta \mathrm{p}$ & 3.23 \\
\hline Hydraulic efficiency [\%] & $\eta_{\mathrm{h}}$ & 32.0 \\
\hline Volumetric efficiency [\%] & $\eta_{\mathrm{v}}$ & 74.0 \\
\hline
\end{tabular}

\section{Experimental setup}

The performances of the pump have been evaluated on a dynamic test bench developed at the University of L'Aquila (Fig. 8). The tested pump has been mechanically actuated through a three-phase DC electric motor driven by an inverter. The motor is able to cover a large range of speeds, ranging to $12000 \mathrm{RPM}$. Through a torque meter the overall mechanical efficiency of the pump can be calculated.

The hydraulic circuit is composed by a 24 liters pressurized tank, which gives the right hydraulic head to the pump; pressure level in the reserve tank can be increased using compressed air, in order to avoid cavitation phenomena during tests. In this way the pressurized engine cooling circuit can be fully simulated. The pump delivers a $50 \%$ glycolwater mixture in the circuit. Flow rate has been measured with a magnetic turbine flow meter, while a proportional pneumatic valve can be regulated to give a specific pressure rise to the circuit, imposing variable pressure head on the pump. Two pressure sensors have been installed at inlet and outlet pipes, to measure the exact pressure drop given by the pump. Input and output signals have been managed in a data acquisition and control unit (DAQ). The components of the test bench and the related uncertainties have been reported in Table 3 . The high value of the mechanical power uncertainty $(<7.0 \%)$ is due to the torque uncertainty, which is constant and independent on the actual measured value. 


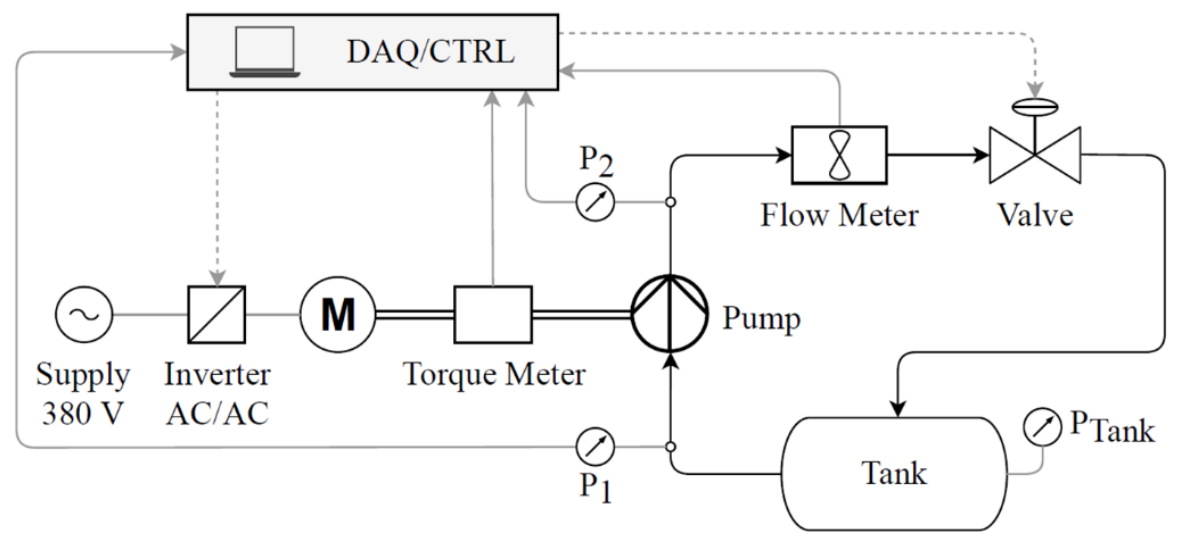

Fig. 8. Test bench layout.

Table 3. Uncertainties on measures.

\begin{aligned} & \hline \multicolumn{2}{c}{ Direct measures } \\ & \hline Torque $0.015 \mathrm{Nm} \\ &$ Velocity $0.01 \%$ of the measured value \\ & Flow rate $0.15 \%$ of the measured value \\ & Pressure $0.003 \mathrm{bar} \\ &$\hline Indirect measures \\ & Pressure rise $<1.5 \% \\ &$ Mechanical power $<7.0 \% \\ &$\hline\end{aligned}

\section{Experimental results}

The designed pump has been built, and it appears as in Fig. 9. The evaluation of the performances of the pump has been realized on the dynamic test bench by measuring flow rate, pressure rise, rotational speed and absorbed torque. The pump has been tested in a range of speed ranging from 8000 RPM up to 12000 RPM. For each speed, seven working points have been acquired, corresponding at seven different valve closure percentages, i.e., circuit pressure drop. 


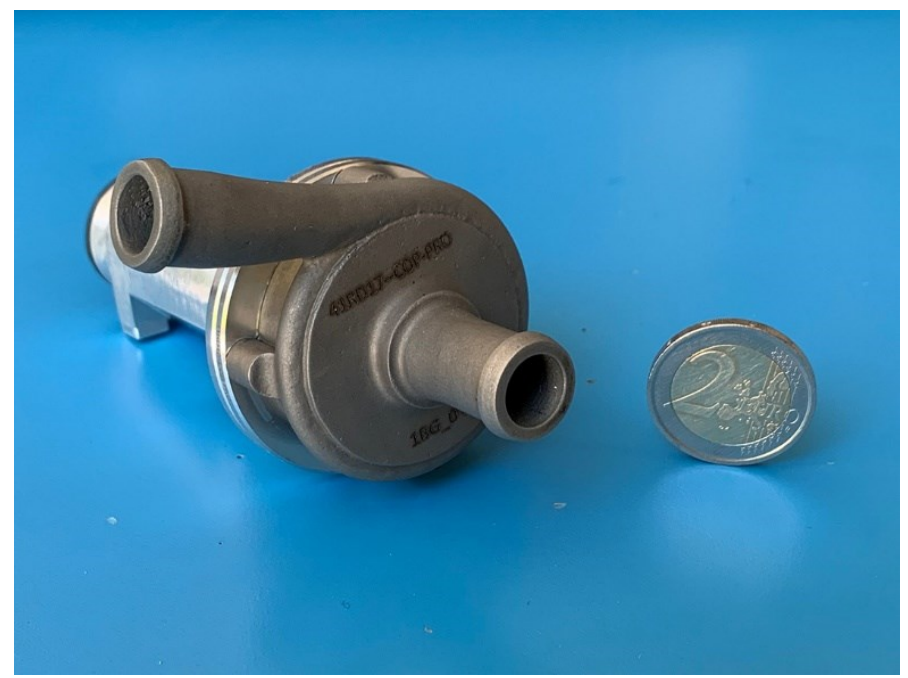

Fig. 9. Pump prototype.

In Fig. 10. Pump dimensionless performances map. the pump performances map, in terms of Q vs. $\Delta \mathrm{p}$, is shown. On the map, the BEP (Best Efficiency Point) of the pump is about $48.2 \%$, located on the $12000 \mathrm{RPM}$ curve, with a flow rate of $14.05 \mathrm{~L} / \mathrm{min}$ and a pressure raise of 2.58 bar, very close to the design point. The efficiency keeps still high on off-design conditions, that are typical for motorsport applications.

On the map, the wide region where efficiency is higher than $90 \%$ of the BEP efficiency includes working point having off-design flow rate and head with deviations of about $60 \%$ of the design conditions. 


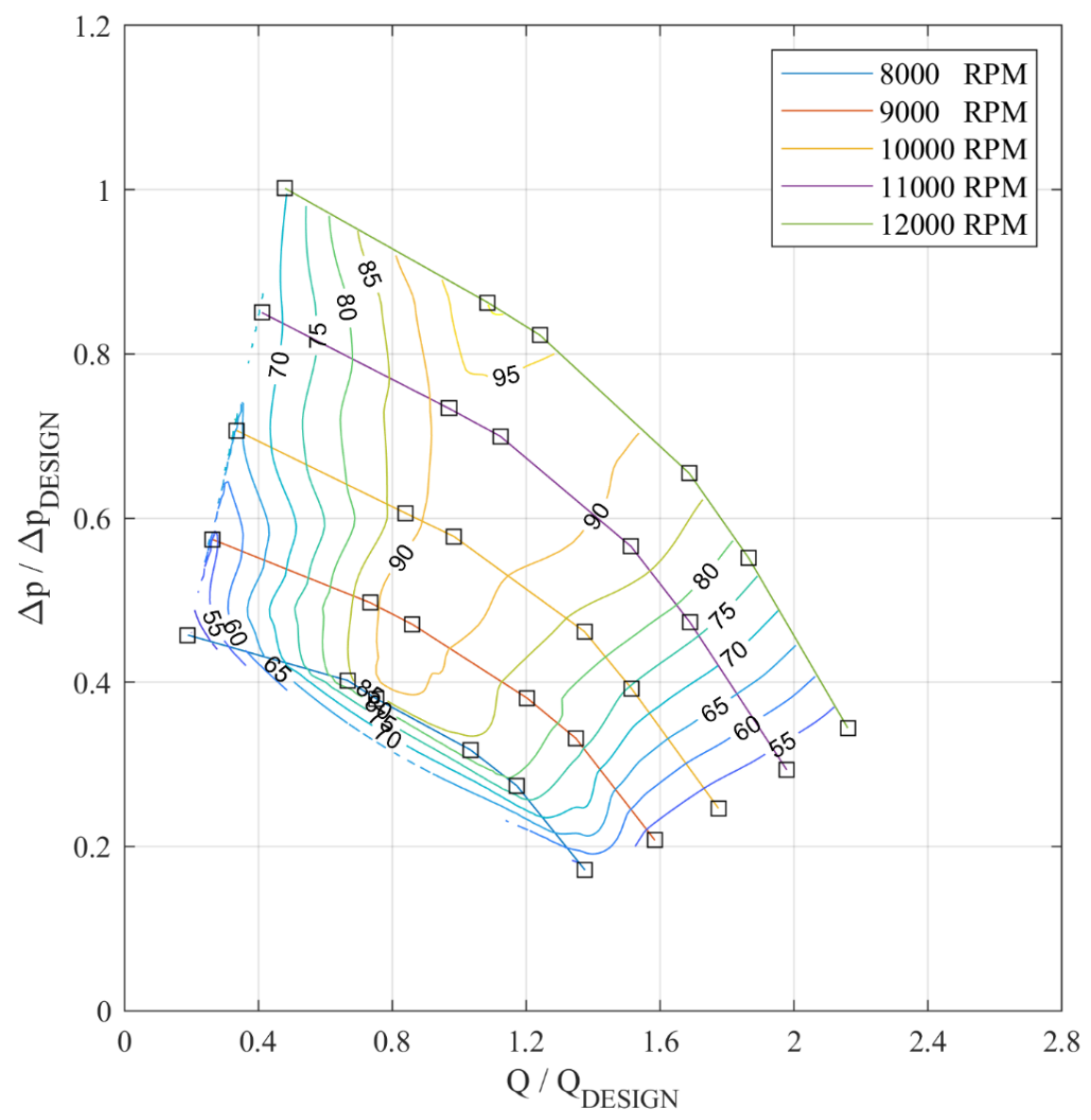

Fig. 10. Pump dimensionless performances map. Black squares are the experimental data acquired, while the iso-contour lines represent the dimensionless efficiency, calculated with respect of BEP efficiency.

One typical issue in pumps designed for high-speed applications, is the power absorbed on bearings and sealing, that forces the mechanical efficiency to quickly decrease in offdesign conditions for lower values of flow rate and pressure delivered, i.e., for low hydraulic power given to the flow.

Moreover, a critical aspect of this kind of pump is that of volumetric losses, which occur on the clearances between pump casing and impeller shroud. Fig. 11 shows how the volumetric efficiency varies with the pump speed, for a set of operating points which represent the cooling circuit, in terms of flow rate and head. The black curve is the theoretical flow rate delivered be the impeller, numerically calculated through the CFD analysis. It has a linear dependence with the pump speed. The blue curve is the experimental flow rate on the same working points. At last, the red curve is the volumetric efficiency of the pump, which ranges from $74 \%$ to $82 \%$. It has been obtained by the ratio between the experimental real flow rate and the numerical flow rate. It is evident how backflows become significant for this kind of pumps; in fact, obtained results correspond to very low values if compared to that of a more conventional centrifugal pump, which lays easily above $90 \%$ [24]. The strong 
effect of volumetric efficiency invites to reduce the clearances if higher overall performances are desired.

Even though the effects on the volumetric efficiency of the pump were expected (due to the reduced dimensions of the pump) the paper offers a quantitative estimation of this phenomenon which can be considered as reference for these operating ranges and pump dimensions, properly modifying the expectations of the engine designer.

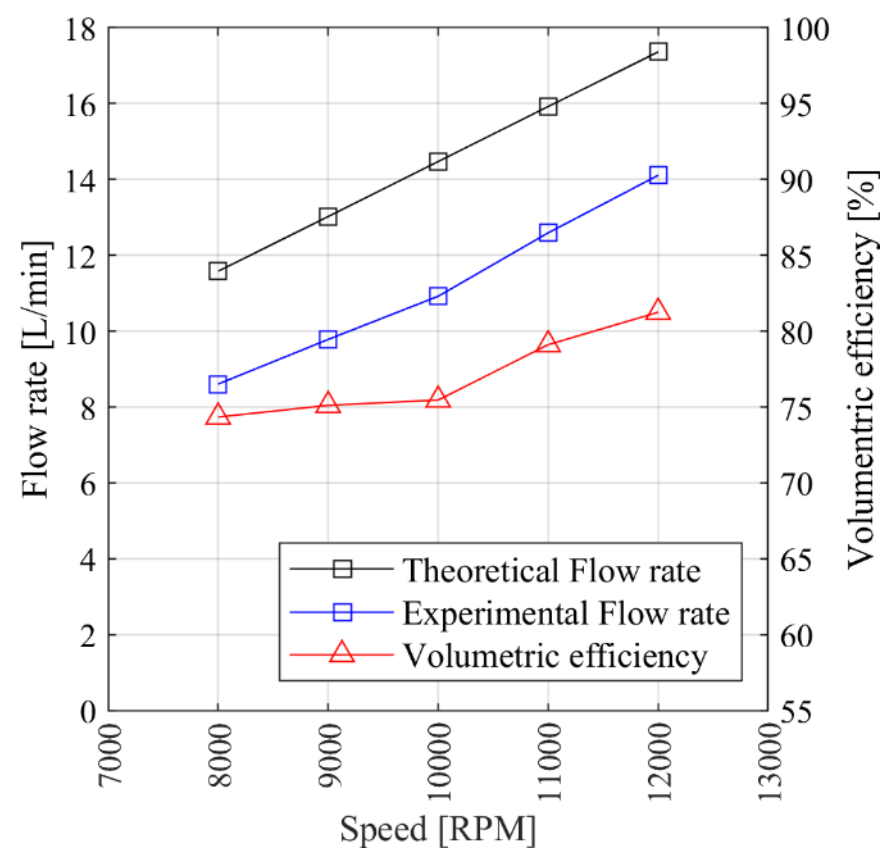

Fig. 11. Volumetric efficiency vs. rotational speed for a set of working point corresponding to the cooling circuit hydraulic resistance.

\section{Conclusions}

In this paper, the design of a high-speed centrifugal pump for the motorsport sector has been discussed. A model-based procedure has been presented to optimize the geometry in the early design phase, through the evaluation of volumetric and fluid dynamic losses and, thus, the estimation of the performances. This allowed to derive a preliminary geometry for the impeller and the volute. Therefore, the design has been verified through CFD analyses. Finally, the pump has been prototyped and tested in a specific test bench. The dimensions obtained are really reduced: not more than $64 \mathrm{~mm}$ as overall maximum encumbrances, as the impeller external diameter is $34 \mathrm{~mm}$, having chosen 12000 RPM as revolution speed design value as the result of a trade-off analysis between dimensions and revolution speed. The maximum efficiency achieved is equal to $48.2 \%$, which is a very high value considering the size of the component and the limiting factors related to impeller head to gap ratio and the afore mentioned volumetric efficiency. A very good off-design conditions are reached which are of interests in the engine cooling applications.

Through theoretical and experimental analyses, main differences with respect to conventional designs have been outlined. They are: 
a) backflow at clearances between pump casing and impeller causes volumetric losses, being the blade outer height similar to the dimension of the gap. The prototype tested has a volumetric efficiency in the range of $75-80 \%$, which significantly reduces the global efficiency.

b) when the pump is driven at very high speeds up to 12000 RPM, cavitation occurs which extends to a sensible part of the inlet section; only a suitable pressurization of the circuit can solve the problem.

c) the organic losses due to the sealing system and shaft support (bearing) are significant mainly at off design conditions when the hydraulic power is reduced.

\section{Acknowledgments}

The paper has been developed in the framework of the Project "Hyper - Hybrid Propulsion for Electric Realignment” - POR FESR 2014-2020 - Call HUB Ricerca e Innovazione.

\section{References}

1. J. S. Pereira, J. B. Ribeiro, R. Mendes, G. C. Vaz, and J. C. André, “ORC based microcogeneration systems for residential application - A state of the art review and current challenges," Renew. Sustain. Energy Rev., vol. 92, no. May, pp. 728-743, 2018, doi: 10.1016/j.rser.2018.04.039.

2. S. F. ul H. Gilani, M. H. bin M. Khir, R. Ibrahim, E. ul H. Kirmani, and S. I. ul H. Gilani, "Modelling and development of a vibration-based electromagnetic energy harvester for industrial centrifugal pump application," Microelectronics J., vol. 66, no. April, pp. 103-111, 2017, doi: 10.1016/j.mejo.2017.06.005.

3. Cipollone, R., Bianchi, G., Di Battista, D., Fatigati, F., Fuel economy benefits of a new engine cooling pump based on sliding vane technology with variable eccentricity, (2015) Energy Procedia, 82, pp. 265-272.

4. Castiglione, T., Pizzonia, F., Bova, S., A novel cooling system control strategy for internal combustion engines, (2016) SAE International Journal of Materials and Manufacturing, 9 (2), pp. 294-302.

5. Cipollone, R., Di Battista, D., Gualtieri, A., A novel engine cooling system with two circuits operating at different temperatures, (2013) Energy Conversion and Management, 75, pp. 581-592.

6. Malvicino, C., Di Sciullo, F., Ferraris, W., Vestrelli, F., Beltramelli, F., Advanced Dual Level Vehicle Heat Rejection System for Passenger Cars, (2012) SAE International Journal of Engines, 5 (3), pp. 1260-1267.

7. Cipollone, R., Di Battista, D., Gualtieri, A., Head and block split cooling in ICE, (2012) IFAC Proceedings Volumes (IFAC-PapersOnline), 45 (30), pp. 400-407.

8. Di Battista, D., Di Bartolomeo, M., Cipollone, R., Flow and thermal management of engine intake air for fuel and emissions saving, (2018) Energy Conversion and Management, 173, pp. 46-55.

9. J. R. Serrano, P. Piqueras, J. De la Morena, A. Gómez-Vilanova, and S. Guilain, "Methodological analysis of variable geometry turbine technology impact on the 
performance of highly downsized spark-ignition engines," Energy, vol. 215, 2021, doi: 10.1016/j.energy.2020.119122.

10. Di Battista, D., Cipollone, R., High efficiency air conditioning model based analysis for the automotive sector, (2016) International Journal of Refrigeration, 64, pp. 108122.

11. J. Guo and F. Jiang, "A novel electric vehicle thermal management system based on cooling and heating of batteries by refrigerant," Energy Convers. Manag., vol. 237, p. 114145, 2021, doi: 10.1016/j.enconman.2021.114145.

12. D. Cha, W. Yang, and Y. Kim, "Performance improvement of self-humidifying PEM fuel cells using water injection at various start-up conditions," Energy, vol. 183, pp. 514-524, 2019, doi: 10.1016/j.energy.2019.06.154.

13. S. Liu, J. Song, J. Shi, and B. Yang, "An improved series-parallel optimization approach for cooling water system," Appl. Therm. Eng., vol. 154, no. February, pp. 368-379, 2019, doi: 10.1016/j.applthermaleng.2019.03.048.

14. A Protopopov and V Vigovskij, Development of methods for high-speed centrifugal pump analysis 2019 IOP Conf. Ser.: Mater. Sci. Eng. 492012003

15. Kraeva, E.M. Optimization of the centrifugal impeller flow passage in high-speed pumps. Russ. Aeronaut. 54, 154 (2011). https://doi.org/10.3103/S1068799811020073

16. Huan, Yy., Liu, Yy., Li, Xj. et al. Experimental and numerical investigations of cavitation evolution in a high-speed centrifugal pump with inducer. $J$ Hydrodyn 33, 140-149 (2021). https://doi.org/10.1007/s42241-021-0006-z

17. Subroto, and Marwan Effendy, Optimization of centrifugal pump performance with various blade number, AIP Conference Proceedings 2114, 020016 (2019); https://doi.org/10.1063/1.5112400

18. Wang W, Li Z. Influence of different types of volutes on centrifugal aviation fuel pump. Advances in Mechanical Engineering. March 2021. doi:10.1177/16878140211005202

19. Xiaoyu Li, Yunguang Ji, Hongbin Cui and Shuqi Xue, Study of Mechanical Performance Affecting Factors in Split Casing Pump. (2020). Regular Issue, 10(2), 31â€“"37. doi:10.35940/ijeat.b1992.1210220

20. Yang Y, Zhou L, Zhou H, Lv W, Wang J, Shi W, He Z. Optimal Design of Slit Impeller for Low Specific Speed Centrifugal Pump Based on Orthogonal Test. Journal of Marine Science and Engineering. 2021; 9(2):121. https://doi.org/10.3390/jmse9020121

21. Mariani, L., Di Bartolomeo, M., Di Battista, D., Cipollone, R., Fremondi, F., Roveglia, R., Experimental and numerical analyses to improve the design of engine coolant pumps, (2020) E3S Web of Conferences, 197, art. no. 06017.

22. R. Cipollone, Viscous Flow Calculation in a centrifugal Impeller, Master Thesis at Von Karman Institute for Fluid dynamics, Brussels 1984

23. R. Cipollone, R.A. Van den Braembussche, Quasi three-dimensional viscous flow in a centrifugal compressor impeller. XXXIX Congresso nazionale dell'Associazione Termotecnica Italiana A.T.I., L'Aquila, Italy, 12-14 September, 1984, Proceedings Vol. II, pp. 971-984. 
24. Lei, C., Yiyang, Z., Zhengwei, W., Yexiang, X., and Ruixiang, L. (July 1, 2015). "Effect of Axial Clearance on the Efficiency of a Shrouded Centrifugal Pump." ASME. J. Fluids Eng. July 2015; 137(7): 071101

25. B. Neumann, "The interaction between geometry and performance of a centrifugal pump," 1991. 